\title{
Analysis of Cathodizing of Anode in Electrogalvanizing Line by Finite Element Method
}

\author{
Masatsugu Morimitsu*, Huimin MEnG, Toshiro Kume, Morio MatsunaGa \\ and Ryuichi OTOGAWA ${ }^{\dagger}$
}

Received June 23, 1997 ; Accepted July 22, 1997

\section{INTRODUCTION}

Insoluble anodes consisting of a metal-oxides coating on a valve metal substrate have been used in electrogalvanizing lines (EGL), 2). It is known that these insoluble anodes deteriorate in the EGL, where the zinc electroplating is done for several sizes of strip $^{3,4)}$. Serious damage of the anode has been appeared with the deposition of zinc, which suggests a partial current reversal on the anode. We call this phenomenon a cathodizing of the anode, which means that a cathodic current flows on a part of the anode. The reasons for the cathodizing are examined from primary potential and current distributions in electroplating cells. The effects of an insulator inserted into the cell are also discussed.

\section{CALCULATION}

The primary potential distribution was calculated for the cross section vertical to the direction of the strip flow in the cell. Since the potential difference between two anodes might be one reason for the cathodizing, we ignored a concentration gradient and a surface overpotential in this study. The primary potential and current distributions in a rectangular coordinate can be determined by Laplace's equation and $\mathrm{Ohm}$ 's law as follows ${ }^{5)}$,

$$
\partial^{2} \phi / \partial^{2} x+\partial^{2} \phi / \partial^{2} y=0
$$

Department of Applied Chemistry, Kyushu Institute of Technology, (Sensui, Tobata, Kitakyushu 804, Japan) $\dagger$ Fine Chemicals Department, Daiso Co., Ltd. (9 Otakasu-cho, Amagasaki 660, Japan)

Key Words: Cathodizing of Anode, Electrogalvanizing Line, Primary Current Distribution, Finite Element Method

$$
i=-\kappa(\partial \phi / \partial n)
$$

where $\kappa$ and $n$ are a conductivity and a unit normal vector perpendicular to electrodes. The boundary condition for the cell as shown in Fig. 1 is given in the following equations,

$$
\begin{array}{ll}
\phi_{t a}=\phi_{1}, \quad \phi_{b a}=\phi_{2} \quad \text { for anodes } \\
\phi_{c}=0 & \text { for cathode } \\
\partial \phi / \partial n=0 & \text { for insulating boundary }
\end{array}
$$

where the subscripts $t a, b a$, and $c$ denote top anode, bottom anode, and cathode. The primary potential and current distributions were simulated by a finite element method using commercial software (Macsyma, PDEase 2).

\section{RESULTS AND DISCUSSION}

Figure 2 shows the typical potential distribution simulated for the cross section of the cell. When the potential of the top anode, $\phi_{1}$, is the same as that of the bottom cathode, $\phi_{2}$, the equipotential lines are symmetrical with respect to the cathode as shown in

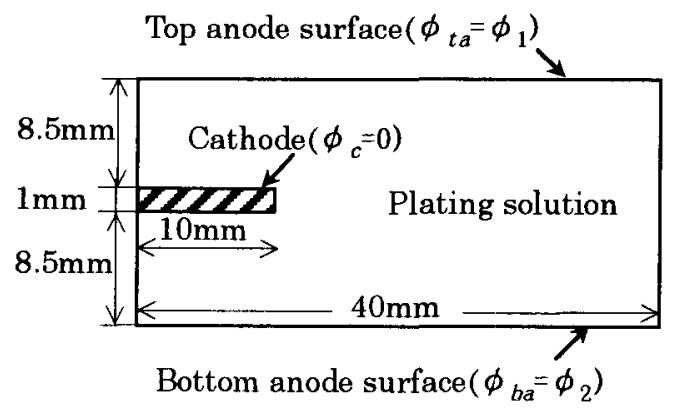

Fig. 1 Schematic drawing of a cell for calculations. 


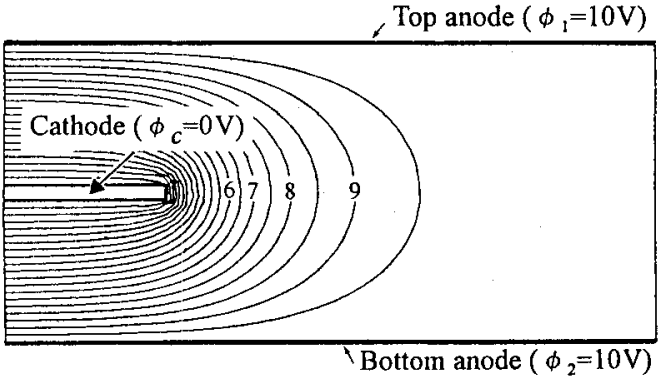

(a)

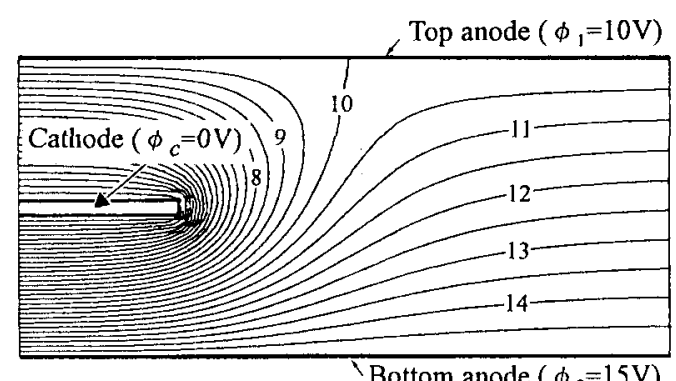

(b)

Fig. 2 Primary potential distributions in the cell.

(a) $\phi_{1}=\phi_{2}=10 \mathrm{~V}$, (b) $\phi_{1}=10 \mathrm{~V}, \phi_{2}=15 \mathrm{~V}$. Curves designate $0.5 \mathrm{~V}$ pitch equipotential surfaces.

Fig. 2a. This potential distribution means that no cathodic current is observed on both anodes. When the potentials of two anodes are different, the equipotential lines become unsymmetrical and the higher equipotential lines in the plating solution are almost parallel to the anodes. This result indicates that the electrolytic current flows directly between two anodes, which causes the cathodic polarization on the anode with lower potential. The total current at the top anode is surely anodic even under this condition. The current reversal occurs at the particular part of the top anode, where no cathode lies between two anodes.

The strip width is shorter than that of the anode in the practical EGL. An insulator, called as an edge mask, is often inserted along the strip edge between the anodes in order to suppress the direct current flow between two anodes. The potential distribution in the cell equipped with the edge mask was, also, simulated and the effects of the shape of the edge mask and the gap between the strip and the edge mask were discussed. As the result shown in Fig. 3, the use of the edge mask can prevent the electrolytic current

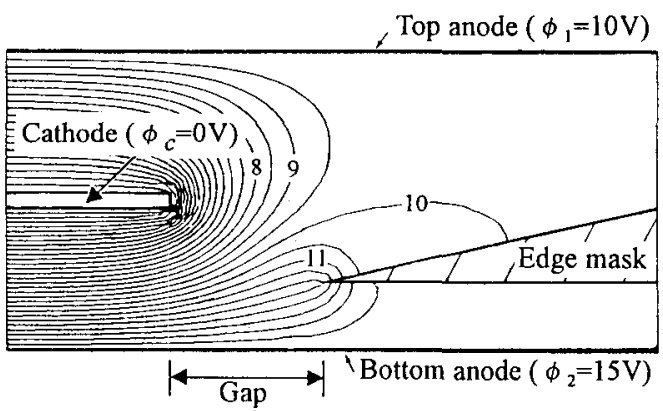

Fig. 3 Primary potential distribution in the cell with edge mask. Gap $=8 \mathrm{~mm}, \phi_{1}=10 \mathrm{~V}, \phi_{2}=15 \mathrm{~V}$. Curves designate $0.5 \mathrm{~V}$ pitch equipotential surfaces.

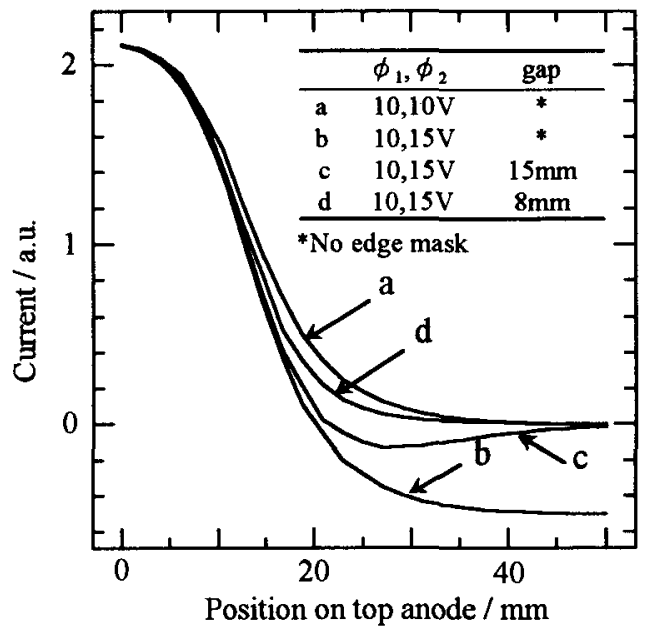

Fig. 4 Current distributions on the top anode.

between two anodes. The proper shape of the edge mask and the shortening the gap between the strip and the edge mask are required to inhibit the cathodizing.

Figure 4 depicts the current distribution on the surface of the top anode in some cells. When the potentials of two anodes are different in the cell without the edge mask (curve b in Fig. 4), the current on the top anode becomes negative at the part where two anodes face without the cathode. As for the curve $\mathrm{b}$, the position on the top anode, at which the current reversal occurs, is $20 \mathrm{~mm}$ as shown in Fig. 4. This position corresponds to the point, at which the equipotential surface line of the same potential as the top anode (10V) reaches the top anode surface in Fig. $2 \mathrm{~b}$. At the region of the top anode where the cathodic current flows in Fig. 4, zinc can be electroplated even 
on the anode.

By comparing the curve $b$ with the curves $c$ and $\mathrm{d}$ in Fig. 4, it is clear that the edge mask can suppress the cathodic polarization on the top anode and the cathodic current disappears by decreasing the gap between the cathode (strip) and the edge mask. The distance of the gap is, however, limited to avoid the contact of the edge mask with the high-speed strip in the practical cell. Therefore, the shape of the edge mask will be important to prevent the cathodizing of the anode.

\section{REFERENCES}

1)S. Ban, K. Inaguchi, T. Tanaka and T. Nakada,Kobe Steel Eng. Rep., 38, 75 (1988).

2)Y. Suemitsu, Galvatech '95 Conf. Proc., pp.3-12, Iron and Steel Soc. (1995).

3)G. N. Martelli, R. Ornelas and G. Faita, Electrochim. Acta, 39, 1551 (1994).

4)K. L. Hardee and L. K. Mitchell, 181 st Meeting of the Electrochemical Society, Abstr. 553 (1991).

5)J. S. Newman, Electrochemical System, 2nd Edition, Prentice Hall, NJ, p. 378 (1991). 